\title{
On finite-time output feedback sliding mode control of an elastic multi-motor system
}

\author{
Pham Tuan Thanh', Tran Xuan Tinh ${ }^{2}$, Dao Phuong Nam ${ }^{3}$, Dao Sy Luat ${ }^{4}$, Nguyen Hong Quang \\ ${ }^{1,2}$ Le Quy Don University of Technology, Hanoi, Vietnam \\ ${ }^{3}$ Hanoi University of Science and Technology, Hanoi, Vietnam \\ ${ }^{4}$ Dong Nai Technology University, Biên Hòa, Vietnam \\ ${ }^{5}$ Thai Nguyen University of Technology, Thai Nguyen, Vietnam
}

\begin{tabular}{ll}
\hline Article Info & ABSTRACT \\
\cline { 1 - 2 } Article history: & $\begin{array}{l}\text { In this paper, the tracking control scheme is presented using the framework } \\
\text { of finite-time sliding mode control (SMC) law and high-gain observer for } \\
\text { disturbed/uncertain multi-motor driving systems under the consideration } \\
\text { Received Jul 28, 2020 } \\
\text { Revised Jan 15, 2021 } \\
\text { Accepted Feb 15, 2021 }\end{array} \quad \begin{array}{l}\text { estimated in connection with linear matrix inequalities (LMIs). The input } \\
\text { state stability (ISS) of proposed controller was analyzed by Lyapunov } \\
\text { stability theory. Finally, the extensive simulation results are given to validate } \\
\text { the advantages of proposed control design. }\end{array}$ \\
\hline Keywords: &
\end{tabular}

Finite-time control

Linear matrix inequalities

Multi-motor systems

Output feedback control

Sliding mode control

This is an open access article under the $\underline{C C B Y-S A}$ license.

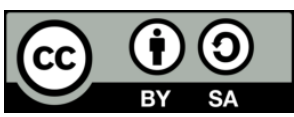

\section{Corresponding Author:}

Nguyen Hong Quang,

Thai Nguyen University of Technology,

666, 3/2 Street, Tich Luong Ward, Thai Nguyen City - Thai Nguyen Province, Vietnam

Email: quang.nguyenhong@tnut.edu.vn

\section{INTRODUCTION}

Multi-motor drive systems have been employed in systems moving paper, metal, material being quite popular in manufacturing systems and researched by many authors in the recent times. The control method utilized artificial neural network (ANN) technique has been presented by Bouchiba et al., [1]. However, the disadvantage is to investigate the appropriate networks with associated learning rules in control design. Furthermore, the effectiveness of tracking problem or the stabilization of the cascade system are not still considered under the influences of using neural network approach. Dominique Knittel, et al., proposed many linear controllers under the consideration of the approximate model of multi-motor systems without elastic, friction as a linear system to design the controller based on the transfer function technique [2], [3]. The framework of the classical PI controller and $\mathrm{H}$ infinity to eliminate disturbance was proposed in the work of [2], [3]. In the elastic multi-motor drive systems, it is necessary to estimate the belt tension to establish the associated state feedback controller. However, the difficulties of the control design lie in the fact that measurement of this belt tension by using sensors. The sliding mode control (SMC) technique based state feedback control enables to eliminate influence of disturbances and unknown parameters was proposed in [1], [4]. In [4], the sliding mode controller was combined with disturbance observer as well as iterative learning control (ILC), feedforward controller. It can be seen that, the classical nonlinear control law has been investigated in robotic systems such as employing the fuzzy-control in inverted pendulum systems [5], robust adaptive control schemes in bilateral teleoperators [6], wheeled mobile robotic systems [7], tractor trailer [8]. 
Additionally, the optimal control and optimization problem are also considered in recent time via the work in [9]-[12]. The sliding mode based control has been paid much attention in recent years because it is a widely relevant control methodology for uncertain/disturbed systems. SMC as a robust control scheme focuses on reducing the disadvantage of external disturbances as much as possible based on the design of appropriate sliding surface as well as implementing the equivalent controller [13]-[21]. An adaptive scheme was proposed in [22] without any knowledge of the bound on the disturbance and their derivatives. The high order SMC design was investigated in control system of unicycle under the consideration of uncertainties of matched and unmatched term [23]. Additionally, the actuator saturation was also considered in sliding mode control design for space craft systems [24]. The problem of investigating state observers for systems is an important direction in the control literature. In [25], high-gain observer (HGO) has been investigated since $1980 \mathrm{~s}$ by the work of Petersen and Hollot on $\mathrm{H}$ infinity control based on the adjusting of appropriate coefficient to satisfy the linear matrix inequalities (LMIs). Authors in [25] described the development from the traditional example to differential observability using Taylor expansion with considering the time interval $[t-T, t]$. It suggested that the HGO should be designed by intermediate variables obtained from the differential observability work [25]. Moreover, in recent time, the HGO design for a class of multi-input multi-output (MIMO) uniformly observable systems were also considered and the extensions of previous idea were implemented for uncertain nonlinear systems with sampled outputs [15]. The work in [26] mentioned two problems, including state estimation via Neural Networks and backstepping technique in dealing with input saturation. It is worth noting that the output feedback control scheme being the framework of state feedback control and observer was designed using dynamic gain and extended state observer [27]. The fixed-time SMC has been mentioned under the consideration of model separation for Dual-Motor Driving systems but the separation technique problem was eliminated [28], [29]. However, most of the previous work in multi-motor drive control systems were not only mentioned to finite time convergence in SMC technique, but also not cared about influence of elastic and friction. Furthermore, observer design for tension was only considered as linear approximate model as well as the separation principle has not been mentioned. In this paper, we consider the LMI based finite-time SMC for multi-motor systems in presence of elastic, friction as well as the high gain observer technique is determined in our work. It is worth noting that the SMC based state feedback control scheme guarantees the elimination of disadvantage of disturbance and uncertainties. Therefore, consideration of using high-gain observer is investigated to compute the tension in this system and combine with the state feedback control scheme to implement the output feedback controller guaranteed the separation principle. The stabilization of cascade system is satisfied by the output feedback control algorithm and shown by theoretical analysis, simulations.

\section{DYNAMIC MODEL OF A ROBOT MANIPULATOR AND PROBLEM STATEMENT}

As the work described in [30], the model of a multi-motor systems with friction, backlash and elastic can be represented as (1):

$$
\left\{\begin{array}{l}
\Delta \dot{\varphi}_{1}=\omega_{r 1}-\omega_{L 1} \\
\Delta \dot{\varphi}_{2}=\omega_{r 2}-\omega_{L 2} \\
\dot{\omega}_{L 1}=\frac{1}{K_{L 1}}\left[k_{c 1} \cdot f_{1}\left(\Delta \varphi_{1}\right)+k_{b 1} g_{1}\left(\Delta \omega_{1}\right)-\left(T_{L 1}+r_{1} F_{21}\right)\right] \\
\dot{\omega}_{L 2}=\frac{1}{K_{L 2}}\left[k_{c 2} \cdot f_{2}\left(\Delta \varphi_{2}\right)+k_{b 2} \cdot g_{2}\left(\Delta \omega_{2}\right)-\left(T_{L 2}-r_{2} F_{12}\right)\right] \\
\dot{F}_{12}=C_{12}\left[r_{1} \omega_{L 1}-r_{2} \omega_{L 2}\left(1+\frac{1}{C_{12} \cdot l} F_{12}\right)\right] \\
\dot{F}_{21}=C_{12}\left[r_{1} \omega_{L 1}-r_{2} \omega_{L 2}\left(1+\frac{1}{C_{12} \cdot l} F_{21}\right)\right] \\
y=F_{12}
\end{array}\right.
$$

In which, the parameters of this system are shown in Table 1. In order to consider the model (1) in the state space representation, the state variables and control inputs are given to obtain the state space model (2):

$$
\begin{aligned}
& x=\left[\begin{array}{llllll}
x_{1} & x_{2} & x_{3} & x_{4} & x_{5} & x_{6}
\end{array}\right]^{T}=\left[\begin{array}{llllll}
\Delta \varphi_{1} & \Delta \varphi_{2} & \omega_{L 1} & \omega_{L 2} & F_{21} & F_{12}
\end{array}\right]^{T} ; \\
& u=\left[\begin{array}{ll}
u_{1} & u_{2}
\end{array}\right]^{T}=\left[\begin{array}{ll}
\omega_{r 1} & \omega_{r 2}
\end{array}\right]^{T} ;
\end{aligned}
$$




$$
\left\{\begin{array}{l}
\dot{x}_{1}=u_{1}-x_{3} ; \dot{x}_{2}=u_{2}-x_{4} \\
\dot{x}_{3}=\frac{1}{K_{L 1}}\left[k_{c 1} \cdot f_{1}\left(x_{1}\right)+k_{b 1} \cdot g_{1}\left(\dot{x}_{1}\right)-\left(T_{L 1}+r_{1} x_{5}\right)\right] \\
\dot{x}_{4}=\frac{1}{K_{L 2}}\left[k_{c 2} \cdot f_{2}\left(x_{2}\right)+k_{b 2} \cdot g_{2}\left(\dot{x}_{2}\right)-\left(T_{L 2}-r_{2} x_{6}\right)\right] \\
\dot{x}_{5}=C_{12}\left[r_{1} x_{3}-r_{2} x_{4}\left(1+\frac{1}{C_{12} \cdot l} x_{5}\right)\right] \\
\dot{x}_{6}=C_{12}\left[r_{1} x_{3}-r_{2} x_{4}\left(1+\frac{1}{C_{12} \cdot l} x_{6}\right)\right]
\end{array}\right.
$$

Table 1. Dynamic parameter

\begin{tabular}{ll}
\hline Paramaters & Explaination \\
\hline $\mathrm{J}_{1}, \mathrm{~J}_{2}, \mathrm{~J}_{\mathrm{L} 1}, \mathrm{~J}_{\mathrm{L} 2}$ & Motors's Inertia moment, Loads's Inertia moment $\left(\mathrm{kgm}^{2}\right)$ \\
$T, T_{L}, \emptyset_{r}$ & The torque of Motor, Load $(\mathrm{Nm})$, rotor's Flux (Wb) \\
$L_{r}$ & Rotor's Self-induction $(\mathrm{H})$ \\
$r, k, \omega_{r}, \omega, F$ & Roller 's Radius, velocity ratio, rotor's electric angle velocity, stator's angle \\
$\omega_{1}, \omega_{2}, \omega_{\mathrm{r} 1}, \omega_{\mathrm{r} 2}$ & velocity, belt tension \\
$\mathrm{c}_{1}, \mathrm{c}_{2}, \mathrm{~b}_{1}, \mathrm{~b}_{2}$ & The angle velocity of motor, load \\
$\Delta \omega_{1}, \Delta \omega_{2}$ & The coefficients of Stiffness and friction \\
\hline
\end{tabular}

Remark 1 : Unlike the description was established in [1]-[4], this work considers multi-motor systems in presence of nonlinear property, backlash, friction, elastic phenomenon. Therefore, the transfer-function based approach in [1]-[4] has not been mentioned in this paper due to these challenges.

The main control objective to find the velocities $\omega_{r 1}, \omega_{r 2}$ obtaining the tracking of state variables vector $x=\left[\Delta \varphi_{1}, \Delta \varphi_{2}, \omega_{L 1}, \omega_{L 2}, F_{21}, F_{12}\right]^{T}$. Furthermore, because it is hard to establish the sensors in this multi-motor system, the controller needs to be added more the state observer obtaining the separation principle.

For the output feedback control design, the assumptions are introduced as follows:

Assumption 1. The diameter of each motorcycle is negligible compared to length of the conveyor belt of a multi-motor system.

Assumption 2. The friction and slip coefficients of the conveyor belt of a multi-motor system are constant and they were not depended on loads, diameter of each motorcycle is negligible compared to length of the conveyor belt of a multi-motor system.

\section{FINITE-TIME OUTPUT FEEDBACK SLIDING MODE CONTROL DESIGN}

\subsection{Finite-time tracking sliding mode control design}

According to the model (3) and Assumption 1, 2, the tracking error model can be obtained as (3):

$$
\left\{\begin{array}{l}
\dot{x}_{1}=C_{12} r_{1} x_{5}-C_{12} r_{2} x_{6}-\frac{r_{2} x_{2} x_{5}}{l} \\
\dot{x}_{2}=C_{12} r_{1} x_{5}-C_{12} r_{2} x_{6}-\frac{r_{2} x_{2} x_{5}}{l} \\
\dot{x}_{3}=u_{1}-x_{5} \\
\dot{x}_{4}=u_{2}-x_{6} \\
\dot{x}_{5}=\frac{1}{K_{L 1}}\left[k_{c 1} \cdot f_{1}\left(x_{3}\right)+k_{b 1} \cdot g_{1}\left(\dot{x}_{3}\right)-\left(T_{L 1}+r_{1} x_{2}\right)\right] \\
\dot{x}_{6}=\frac{1}{K_{L 2}}\left[k_{c 2} \cdot f_{2}\left(x_{4}\right)+k_{b 2} \cdot g_{2}\left(\dot{x}_{4}\right)-\left(T_{L 2}-r_{2} x_{1}\right)\right]
\end{array}\right.
$$

It can be rewritten by: 


$$
\left[\begin{array}{c}
\dot{x}_{1} \\
\dot{x}_{2} \\
\dot{x}_{3} \\
\dot{x}_{4} \\
\dot{x}_{5} \\
\dot{x}_{6}
\end{array}\right]=\left[\begin{array}{cccccc}
0 & 0 & 0 & 0 & C_{12} \cdot r_{1} & -C_{12} \cdot r_{2} \\
0 & 0 & 0 & 0 & C_{12} \cdot r_{1} & -C_{12} \cdot r_{2} \\
0 & 0 & 0 & 0 & -1 & 0 \\
0 & 0 & 0 & 0 & 0 & -1 \\
\frac{-r_{1}}{K_{L 1}} & 0 & 0 & 0 & 0 & 0 \\
0 & \frac{r_{2}}{K_{L 2}} & 0 & 0 & 0 & 0
\end{array}\right] \cdot\left[\begin{array}{c}
x_{1} \\
x_{2} \\
x_{3} \\
x_{4} \\
x_{5} \\
x_{6}
\end{array}\right]+\left[\begin{array}{cccc}
0 & 0 & 0 & 0 \\
0 & 0 & 0 & 0 \\
1 & 0 & 0 & 0 \\
0 & 1 & 0 & 0 \\
0 & 0 & 0 & 0 \\
0 & 0 & 0 & 0
\end{array}\right] \cdot\left[\begin{array}{c}
u_{1} \\
u_{2} \\
0 \\
0
\end{array}\right]+d(\underline{x}, t)
$$

where: $\underline{z}_{1}=\left[\begin{array}{ll}x_{1} & x_{2}\end{array}\right]^{T} ; \underline{z}_{2}=\left[\begin{array}{llll}x_{3} & x_{4} & x_{5} & x_{6}\end{array}\right]^{T} ; A_{11}=\left[\begin{array}{cc}0 & 0 \\ 0 & 0\end{array}\right] ; A_{12}=\left[\begin{array}{cccc}0 & 0 & C_{12} r_{1} & -C_{12} r_{2} \\ 0 & 0 & C_{12} r_{1} & -C_{12} r_{2}\end{array}\right]$

$$
\begin{aligned}
& A_{21}=\left[\begin{array}{cc}
0 & 0 \\
0 & 0 \\
\frac{-r_{1}}{K_{L 1}} & 0 \\
0 & \frac{r_{2}}{K_{L 2}}
\end{array}\right] ; A_{22}=\left[\begin{array}{cccc}
0 & 0 & -1 & 0 \\
0 & 0 & 0 & -1 \\
0 & 0 & 0 & 0 \\
0 & 0 & 0 & 0
\end{array}\right] \\
& B_{2}=\left[\begin{array}{llll}
1 & 0 & 0 & 0 \\
0 & 1 & 0 & 0 \\
0 & 0 & 0 & 0 \\
0 & 0 & 0 & 0
\end{array}\right] ; B_{1}=\left[\begin{array}{llll}
0 & 0 & 0 & 0 \\
0 & 0 & 0 & 0
\end{array}\right] \quad \underline{d}_{2}\left(\underline{z}_{2}, t\right)=\left[\begin{array}{c}
0 \\
0 \\
\frac{1}{K_{L 1}}\left[k_{c 1} \cdot f_{1}\left(x_{3}\right)+k_{b 1} \cdot g_{1}\left(\dot{x}_{3}\right)-T_{L 1}\right] \\
\frac{1}{K_{L 2}}\left[k_{c 2} \cdot f_{2}\left(x_{4}\right)+k_{b 2} \cdot g_{2}\left(\dot{x}_{4}\right)-T_{L 2}\right]
\end{array}\right]
\end{aligned}
$$

therefore, the tracking error model (4) can be represented as:

$$
\left\{\begin{array}{l}
\dot{\underline{z}}_{1}=\left[\begin{array}{cc}
0 & 0 \\
0 & 0
\end{array}\right] \underline{z}_{1}+\left[\begin{array}{llll}
0 & 0 & C_{12} r_{1} & -C_{12} r_{2} \\
0 & 0 & C_{12} r_{1} & -C_{12} r_{2}
\end{array}\right] \underline{z}_{2} \\
{\underline{\underline{z_{2}}}}_{2}=\left[\begin{array}{cc}
0 & 0 \\
0 & 0 \\
\frac{-r_{1}}{K_{L 1}} & 0 \\
0 & \frac{r_{2}}{K_{L 2}}
\end{array}\right] \underline{z}_{1}+\left[\begin{array}{cccc}
0 & 0 & -1 & 0 \\
0 & 0 & 0 & -1 \\
0 & 0 & 0 & 0 \\
0 & 0 & 0 & 0
\end{array}\right] \underline{z}_{2}+B_{2} \underline{u}+\underline{d}_{2}\left(z_{2}, t\right)
\end{array}\right.
$$

Consider the sliding variable $\underline{s}(\underline{e})=\Lambda \underline{e}-A_{12}^{T} P v\left(\underline{e}_{1}\right)$

where $\underline{e}=\underline{\mathrm{z}}^{\text {ref }}-\underline{z}$ and $v\left(e_{1}\right)=-\rho\left|e_{1}\right|^{\eta-1}, \Lambda=\left[\begin{array}{ll}H & I\end{array}\right]$,

the finite time sliding mode control law is proposed as described in the following Theorem 1.

Theorem 1: Consider the system (5) with the disturbance $\left(d_{2}(\underline{z}, t)\right)$ being bounded by $\bar{d}_{2}$ and the SMC is given as;

$$
u(t)=u_{n}(t)-B_{2}^{-1} u_{s}(t)
$$

in which $u_{n}(t)=-B_{2}^{-1}\left(\Lambda A \underline{z}-A_{12}^{T} P \frac{d v\left(\underline{e}_{1}\right)}{d t}-\Lambda \underline{\dot{z}}_{d}\right), u_{s}(t)=\bar{d}_{2} \operatorname{sgn}(s(\underline{e}))+\sigma \operatorname{sgn}(s(\underline{e}))|s(\underline{e})|^{\eta}+\gamma s(\underline{e}), \sigma ; \gamma$ are the positive constant numbers. The proposed SMC enables us to obtain the finite-time Input State Stability (ISS) stability.

Proof : The Proof is divided into two steps. 
In Step 1, we prove that the closed system reaches to the sliding surface in finite time: Based on the Lyapunov candidate function using the sliding variable as:

$$
V_{2}(s(\underline{e}))=\frac{1}{2} s^{T}(\underline{e}) s(\underline{e})
$$

taking the time derivative of this Lyapunov function along the systems (5), we obtain:

$$
\begin{aligned}
\dot{V}_{2}(s(\underline{e})) & =s^{T}(\underline{e}) \dot{s}(\underline{e})=s^{T}(\underline{e})\left(H \dot{z}_{1}-A_{12}^{T} P \frac{d g\left(\underline{e}_{1}\right)}{d t}+A_{21} \underline{z}_{1}+A_{22} \underline{z}_{2}+B_{2} \underline{u}-H \underline{\dot{z}}_{1 d}-\underline{\dot{z}}_{2 d}+d_{2}(\underline{z}, t)\right) \\
& =s^{T}(\underline{e})\left(\Lambda A \underline{z}-A_{12}^{T} P \frac{d g\left(\underline{e}_{1}\right)}{d t}+B_{2} \underline{u}-\Lambda \underline{\dot{z}}_{d}+d_{2}(\underline{z}, t)\right)
\end{aligned}
$$

substituting (7) into (9) gives:

$$
\begin{array}{r}
\dot{V}_{2}(s(\underline{e}))=s^{T}(\underline{e}) \sigma \operatorname{sgn}(s(\underline{e}))|s(\underline{e})|^{\eta}-s^{T}(\underline{e}) \gamma s(\underline{e}) \\
-s^{T}(\underline{e}) \bar{d}_{2} \operatorname{sgn}(s(\underline{e}))+s^{T}(\underline{e}) d_{2}(\underline{z}, t)
\end{array}
$$

according to $\bar{d}_{2} \geq\left(d_{2}(\underline{z}, t)\right)_{\max }$, we have:

$$
\dot{V}_{2}(s(\underline{e})) \leq-\lambda_{\min }(\gamma)\|s(\underline{e})\|^{2}-\lambda_{\min }(\sigma)\|s(\underline{e})\|^{\eta+1}=-\alpha_{2} V_{2}(s(\underline{e}))-\beta_{2} V_{2}^{\eta_{2}}(s(\underline{e}))
$$

Choose $\alpha_{2}=2 \lambda_{\min }(\gamma)>0, \beta_{2}=2^{(\eta+1) / 2} \lambda_{\min }(\sigma)>0, \eta_{2}=(\eta+1) / 2<1$, according to the inequality (11), the closed system reaches to sliding surface in finite time given as (12):

$$
t_{r 2}=\frac{1}{\alpha_{2}\left(1-\eta_{2}\right)} \ln \frac{\alpha_{2} V_{2}\left(s\left(\underline{e}\left(t_{0}\right)\right)\right)^{1-\eta_{2}}+\beta_{2}}{\beta_{2}}
$$

where

$$
V_{2}\left(s\left(\underline{e}\left(t_{0}\right)\right)\right)=(1 / 2) s^{T}\left(\underline{e}\left(t_{0}\right)\right) s\left(\underline{e}\left(t_{0}\right)\right)
$$

In Step 2, once the closed system trajectory reaches the sliding surface, the errors converge to attraction region in finite time. Consider the closed system in the sliding surface $\underline{s}(\underline{e})=\Lambda \underline{e}-A_{12}^{T} P v\left(\underline{e}_{1}\right)=0$. Choose $\bar{\varsigma}$ and $k$ are constant coefficients, in which $\|f(\mathrm{t})\| \leq \bar{\varsigma} ;\|\underline{e}\| \geq k$. The parameters $\rho>0, \mu_{1}>0$ và $\mu_{2}>0$ and the matrices $X>0, Q>0, W>0, G>0$ and $\mathrm{Y}$ are chosen to satisfy the following Linear Matrix Inequalities (LMIs):

$$
\begin{aligned}
& {\left[\begin{array}{cc}
A_{11} X+X A_{11}^{T}-A_{12} Y-Y^{T} A_{12}^{T}+Q & X \\
X & -W
\end{array}\right]<0} \\
& {\left[\begin{array}{cc}
-2 \rho A_{12} A_{12}^{T} & X \\
X & G
\end{array}\right]<0} \\
& \mu_{1} I_{2 \times 2}-Q<0 \\
& \mu_{2} I_{2 \times 2}-W>0 \\
& \bar{c}_{1}-\frac{\varsigma}{k^{2}} \mu_{2}>0
\end{aligned}
$$


Then the system (5) is stabilized in finite time with the attraction region $\left\|e_{1}(t)\right\| \leq k$. The Proof is similarly implemented as above step with the corresponding Lyapunov Function $V_{1}\left(\underline{e}_{1}\right)=\underline{e}_{1}^{T} P \underline{e}_{1}, P=X^{-1}$

\subsection{High-gain observer control design of multi-motor systems}

As described in [25], it is hard to find the observer for multi-output systems because the data collection needs to be implemented in the sufficiently small interval as well as employing the taylor series approximation. Therefore, the high-gain observer was proposed by [15] enabling us to deal with multi-output systems. Consider the class of nonlinear uniformly observable systems:

$$
\left\{\begin{array}{l}
\dot{z}=f(u, z)+\varepsilon \\
y=\bar{C} z=z^{1}
\end{array}\right.
$$

where the state variables $z \in R^{n}$ with $z^{k} \in R^{n_{k}} ; k=1,2,3, \ldots ., q ; n_{1} \geq n_{2} \geq \ldots n_{q} ; \sum_{k=1}^{n} n_{k}=n$, the input $u \subset U$ is a compact set of $\mathrm{R}^{\mathrm{m}}$, the output variables $y \in R^{n_{1}}$.

$$
\begin{aligned}
& z=\left(\begin{array}{l}
z^{1} \\
z^{2} \\
\vdots \\
z^{q}
\end{array}\right) ; f(u, z)=\left(\begin{array}{l}
f_{1}\left(\mathrm{u}, \mathrm{z}^{1}, \mathrm{z}^{2}\right) \\
f_{2}\left(\mathrm{u}, \mathrm{z}^{1}, \mathrm{z}^{2}, \mathrm{z}^{3}\right) \\
\vdots \\
f_{q-1}(\mathrm{u}, \mathrm{z}) \\
0
\end{array}\right) ; \varepsilon=\left(\begin{array}{l}
0 \\
\vdots \\
0 \\
\varepsilon^{q-1} \\
\varepsilon^{q}
\end{array}\right) ; \bar{C}=\left[I_{n_{1}}, 0_{n_{1} \times n_{2}}, \ldots, 0_{n_{1} \times n_{q}}\right] \\
& \bar{C}=\left[I_{n_{1}}, 0_{n_{1} \times n_{2}}, \ldots, 0_{n_{1} \times n_{q}}\right]
\end{aligned}
$$

This above system also satisfies several assumptions as described in [15]. It is hard to find directly the High-Gain observer for model (19), so that it can be transferred into the following form to easier design based on the transformation as follows:

the intermediate observer [15]:

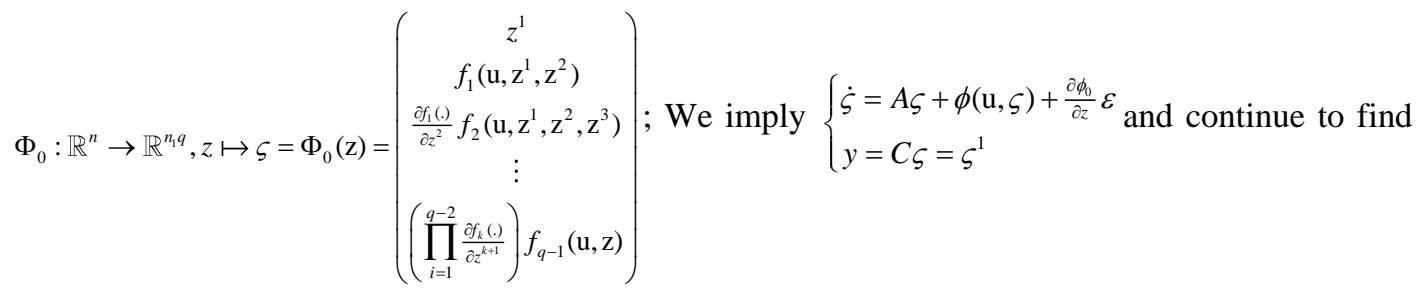

$\dot{\hat{\zeta}}=A \hat{\zeta}+\phi(\mathrm{u}, \hat{\zeta})-\theta \Delta_{\theta}^{-1} S^{-1} C^{T} \tilde{\zeta}^{-1}-\theta \Gamma(\mathrm{u}, \hat{\varsigma}) \Delta_{\theta}^{-1} S^{-1} C^{T} \tilde{\zeta}^{-1}$. Because $\theta \Delta_{0}^{-1} A \Delta_{\theta}=A$ và $C \Delta_{0}=C$ then employing $\bar{\varsigma}=\Delta_{0} \tilde{\varsigma}$, we obtain the dynamic equation of observer errors:

$\dot{\bar{\zeta}}=\theta\left(\mathrm{A}-\mathrm{S}^{-1} \mathrm{C}^{T} \mathrm{C}\right) \bar{\zeta}+\Delta_{\theta}(\phi(\mathrm{u}, \hat{\zeta}))-\Delta_{\theta} \frac{\partial \Phi_{0}}{\partial z} \varepsilon-\theta \Delta_{\theta} \Gamma(\mathrm{u}, \hat{\zeta}) \Delta_{\theta}^{-1} C^{T} \tilde{\varsigma}^{1}$. In order to analyze the observer errors, selecting the candidate function $V_{1}(\bar{\zeta})=\bar{\zeta}^{T} S \bar{\zeta}$, take the derivative of this function along the system trajectory of transformed systems:

$$
\dot{V}_{1} \leq-\theta V_{1}+2 \sigma^{2}(\mathrm{~S})\|\bar{\zeta}\|\left(\zeta\|\bar{\zeta}\|+\frac{\rho}{\lambda_{\min }(\mathrm{s})}\left\|\tilde{\zeta}^{1}\right\|\right)+\left\|\Delta_{0} \frac{\partial \Phi_{0}\left(\mathrm{u}, \Phi_{0}^{c}(\hat{\zeta})\right)}{\partial z} \varepsilon\right\| \text { where } \sigma(\mathrm{S})=\sqrt{\frac{\lambda_{\max }(\mathrm{s})}{\lambda_{\min }(\mathrm{s})}}
$$

based on the work in [15], we imply that:

$$
\dot{V}_{1} \leq-\left(\theta-c_{1}\right) V_{1}+\frac{c_{2}}{\theta^{q-1}} \delta_{\theta} \sqrt{V_{1}}
$$

the fact that is the following selections: $\Delta_{\theta}=\operatorname{blockdiag}\left(\mathrm{I}_{n_{1}}, \frac{1}{\theta} \mathrm{I}_{n_{1}}, \ldots, \frac{1}{\theta^{4-1}} \mathrm{I}_{n_{1}}\right)$ 
$\Lambda^{+}(\hat{\mathrm{z}})$ is the left inverse of block diagonal matrix $\Lambda(\hat{\mathrm{z}})$ with $\Lambda(\hat{\mathrm{z}})=$ blockdiag $\left(I_{n_{1}}, \frac{\partial f_{1}\left(\mathrm{u}, \hat{\mathrm{z}}^{1}, \hat{\mathrm{z}}^{2}\right)}{\partial \hat{z}^{2}}, \ldots, \prod_{i=1}^{q-1} \frac{\partial f_{k}(\mathrm{u}, \hat{\mathrm{z}})}{\partial \hat{z}^{k+1}}\right)$ where $\mathrm{S}$ is the positive definite solution of the algebraic Lyapuov equation (21):

$$
S+A^{T} S+S A-C^{T} C=0
$$

comeback to the model (19), the equivalent observer can be determined as (22):

$$
\dot{\hat{z}}=\left(\frac{\partial \Phi(\hat{\mathrm{z}})}{\partial z}\right)^{+}(\dot{\hat{\zeta}})=\mathrm{f}(\mathrm{u}, \hat{\mathrm{z}})-\theta \Lambda^{+}(\hat{\mathrm{z}}) \Delta_{\theta}^{-1} S^{-1} C^{T} \bar{C}(\hat{\mathrm{z}}-\mathrm{z})
$$

although implementing the observer intermediately, we still obtain the direct result (23):

$$
\theta \Lambda^{+}(\hat{\mathrm{z}}) \Delta_{\theta}^{-1} S^{-1} C^{T}=\left(\begin{array}{c}
\theta C_{1}^{q} I_{n_{1}} \\
\theta^{2} C_{2}^{q}\left[\frac{\partial f_{1}}{\partial x^{2}}(\mathrm{u}, \mathrm{z})\right]^{+} \\
\vdots \\
\theta^{q} C_{q}^{q}\left[\prod_{i=1}^{q-1} \frac{\partial f_{k}}{\partial z^{k+1}}(\mathrm{u}, \mathrm{z})\right]^{+}
\end{array}\right)
$$

where $S(\mathrm{i}, \mathrm{j})=(-1)^{i+j} C_{i+j-2}^{j-1} I_{n_{1}}$ with $C_{j}^{i}=\frac{j !}{i !(\mathrm{j}-\mathrm{i}) !} ; 1 \leq \mathrm{i}, \mathrm{j} \leq \mathrm{q}$

in order to find control design appropriate HGO for multi-motor systems (1), we need to implement alternately:

Consider the following multi-motor systems:

$$
\left\{\begin{array}{l}
\dot{x}_{1}=\frac{1}{T}\left(u-x_{2}\right) \\
\dot{x}_{2}=J_{L}\left[\frac{1}{K_{T C}} f_{1}\left(x_{1}\right)+\mathrm{K}_{C}\left(u-x_{2}\right) f_{2}\left(x_{1}\right)-\left(\mathrm{T}_{L}+r \cdot x_{3}\right)\right] \\
\dot{x}_{3}=C_{12}\left[r_{1} x_{21}-r_{2} x_{22}\left(1+\frac{1}{C_{12} \cdot l} x_{3}\right)\right] \\
y=x_{3}
\end{array}\right.
$$

where

$$
x_{1}=\left[\begin{array}{l}
x_{11} \\
x_{12}
\end{array}\right]=\left[\begin{array}{l}
\Delta \varphi_{1} \\
\Delta \varphi_{2}
\end{array}\right] \in \mathbb{R}^{2} ; \quad x_{2}=\left[\begin{array}{l}
x_{21} \\
x_{22}
\end{array}\right]=\left[\begin{array}{l}
\omega_{r 1} \\
\omega_{r 2}
\end{array}\right] \in \mathbb{R}^{2} ; x_{3}=\left[\begin{array}{c}
x_{31} \\
x_{32}
\end{array}\right]=\left[\begin{array}{l}
F_{21} \\
F_{12}
\end{array}\right] \in \mathbb{R}^{2}
$$

according to the result (22), we obtain the equivalent observer:

$$
\left\{\begin{array}{l}
\dot{\hat{x}}_{1}=\frac{1}{T}\left(\mathrm{u}-\hat{\mathrm{x}}_{2}\right)-3 \theta\left(\hat{\mathrm{x}}_{3}-x_{3}\right) \\
\dot{\hat{x}}_{2}=J_{L}\left[\frac{1}{K_{T C}} f_{1}\left(\hat{x}_{1}\right)+\mathrm{K}_{C}\left(u-\hat{x}_{2}\right) f_{2}\left(\hat{x}_{1}\right)-\left(\mathrm{T}_{L}+r \cdot \hat{x}_{3}\right)\right]+\frac{\theta^{2}}{T}\left(\hat{x}_{3}-x_{3}\right) \\
\dot{\hat{x}}_{3}=C_{12}\left[r_{1} \hat{x}_{21}-r_{2} \hat{x}_{22}\left(1+\frac{1}{C_{12} \cdot l} \hat{x}_{3}\right)\right]+r J_{L} \theta^{3}\left(\hat{x}_{3}-x_{3}\right) \\
\mathrm{y}=x_{3}
\end{array}\right.
$$




\section{OFFLINE SIMULATION RESULTS}

To clearly validate the efficacy of the proposed output feedback control scheme, a multi-motor driving system is established in Figure 1, which shows the physical meaning of parameters as well as the completed control system. The simulation results in Figures 2 and 3 describe the behavior of tension and velocities when the load is changed from $T_{L 1}=100 \mathrm{Nm}$ to $T_{L 2}=50 \mathrm{Nm}$. It should be noted that the comparison between the response using proposed solution and the classical approaches has been investigated. Furthermore, the simulation results in Figure 4 show the response of proposed high gain observer in MultiMotor systems.

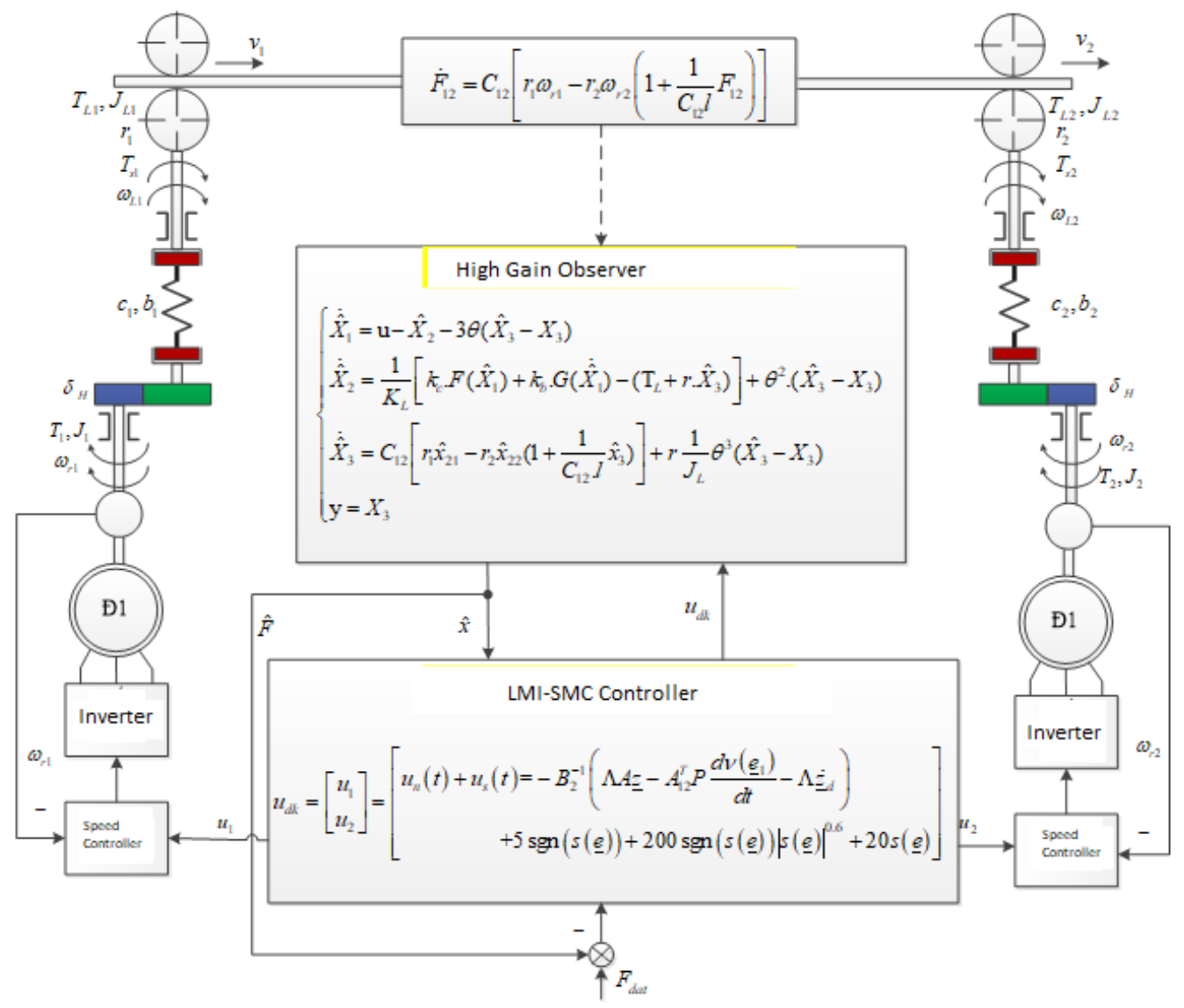

Figure 1. The structure of multi-motor control system

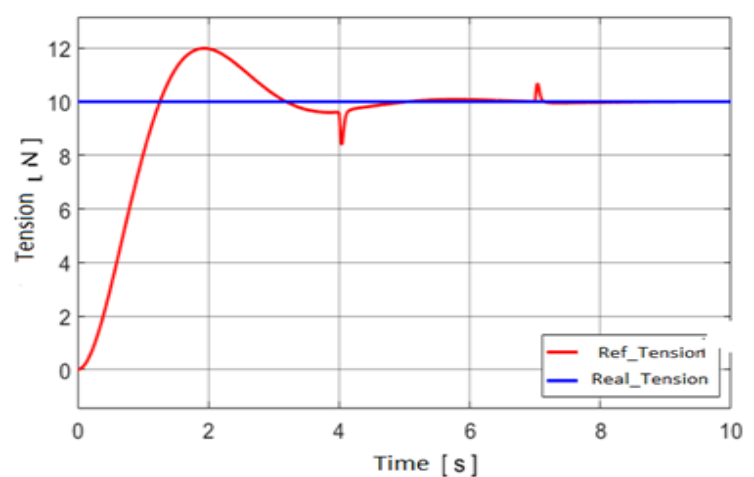

(a)

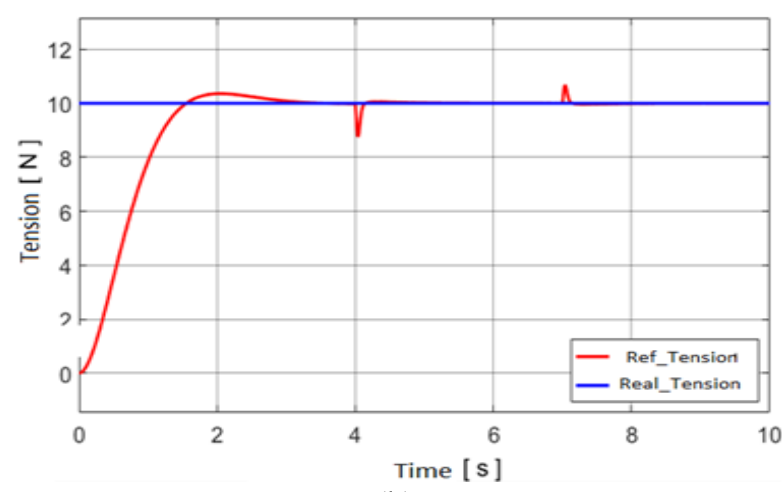

(b)

Figure 2. (a) The response of tension PID with the load variation (b) The response of tension LMI-SMC with the load variation 


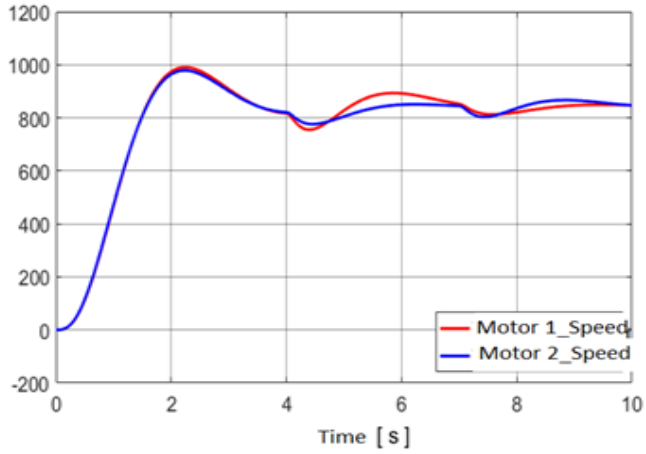

(a)

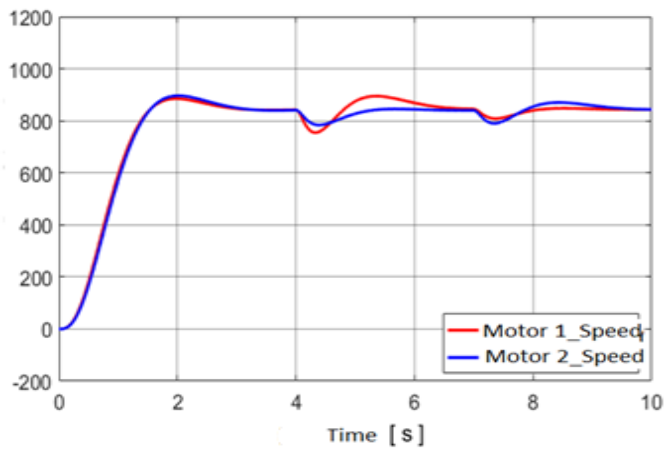

(b)

Figure 3. (a) The response of speed PID with the load variation (b) The response of speed LMI-SMC with the load variation

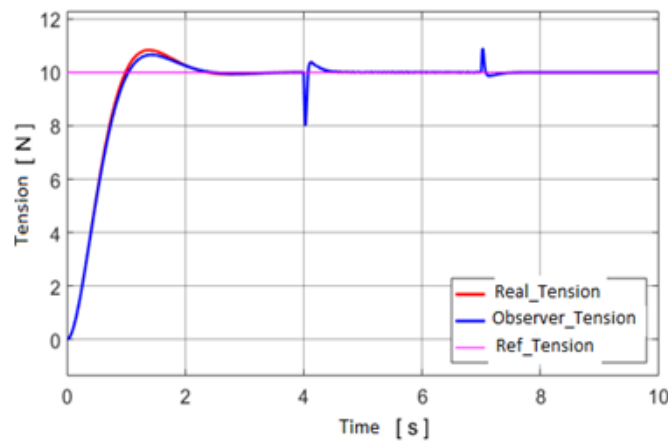

(a)

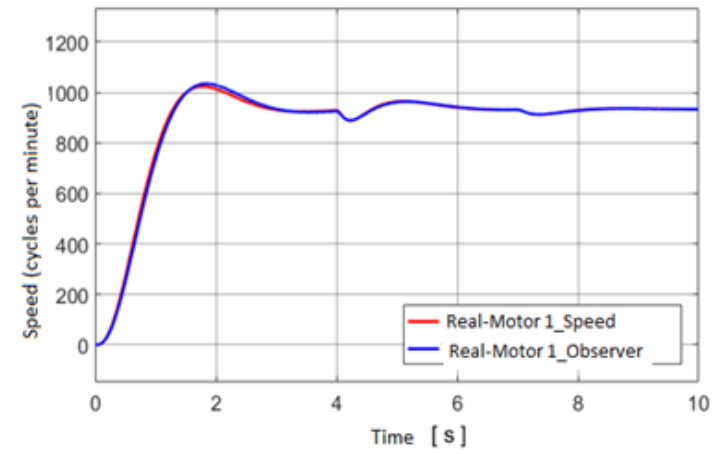

(b)

Figure 4. The response of observer, (a) tension observer, (b) speed Observer, with gear gap $\alpha=0.15 \mathrm{rad}$ $\left(8,59^{0}\right)$

\section{CONCLUSION}

To deal with the multi-motor systems in presence of nonlinear property, backlash, elastic, the finite time sliding mode controller combining with high gain observer is developed in this work. The convergence time of SMC is clearly estimated by LMI technique and the observer is dealt with multi-output systems. The proposed controller is validated via simulations in comparison with existing solution.

\section{ACKNOWLEDGEMENTS}

This research was supported by Research Foundation funded by Thai Nguyen University of Technology.

\section{REFERENCES}

[1] Bousmaha Bouchiba, Ismail Khalil Bousserhane, Mohammed Karim Fellah, Hazzab, "Neural Network Sliding Mode Control for Multi-Machine Web Winding System," Revue Roumaine des Sciences Techniques - Serie Électrotechnique et Énergétique, vol. 62, no. 1, pp. 109-113, 2017.

[2] Hakan Koc, Dominique Knittel, Michel de Mathelin, and Gabriel Abba, "Modeling and Robust Control of Winding Systems for Elastic Webs," IEEE Transaction on Control Systems Technology, vol. 10, no. 2, pp. 197-208, 2002.

[3] Dominique Knittel, Edouard Laroche, Daniel Gigan, and Hakan Koç, "Tension Control for Winding Systems With Two-Degrees-of-Freedom Hinf Controllers," IEEE Transactions on Industry Application, vol. 39, no. 1, pp. 113$120,2003$.

[4] Jie-Shiou Lu, Ming-Yang Cheng, Ke-Han Su, Mi-Chi Tsai, "Wire tension control of an automatic motor winding machine an iterative learning sliding mode control approach," Robotics and Computer-Integrated Manufacturing, vol. 50, pp. 50-62, 2018. 
[5] Mohammed A. A. Al-Mekhlafi, Herman Wahid, Azian Abd Aziz, "Adaptive Neuro-Fuzzy Control Approach for a Single Inverted Pendulum System," International Journal of Electrical and Computer Engineering (IJECE), vol. 8, no. 5, pp. 3657-3665, 2018.

[6] Liu Y. C., Dao N., Zhao K. Y., "On Robust Control of Nonlinear Teleoperators under Dynamic Uncertainties with Variable Time Delays and without Relative Velocity," IEEE Transactions on Industrial Informatics, vol. 16, no. 2, pp. 1272-1280, 2019.

[7] Nguyena, T., Hoang, T., Pham, M., \& Dao, N., "A Gaussian wavelet network-based robust adaptive tracking controller for a wheeled mobile robot with unknown wheel slips," International Journal of Control, vol. 92, no. 11, pp. 2681-2692, 2019.

[8] Binh, N. T., Tung, N. A., Nam, D. P., Quang, N. H., "An adaptive backstepping trajectory tracking control of a tractor trailer wheeled mobile robot," International Journal of Control, Automation and Systems, vol. 17, no. 2, pp. 465-473, 2019.

[9] Dwi Prihanto, Irawan Dwi Wahyono, Suwasono and Andrew Nafalski, "Virtual Laboratory for Line Follower Robot Competition," International Journal of Electrical and Computer Engineering (IJECE), vol. 7, no. 4, pp. 2253-2260, 2017.

[10] Phuong Nam Dao, Hong Quang Nguyen, Phuong Nam Tran, Hai Yen Tran Thi, “Adaptive Dynamic Programing based Optimal Control for a Robot Manipulator," International Journal of Power Electronics and Electrical Drives (IJPEDS), vol. 11, no. 3, pp. 1123-1131, 2020.

[11] Nam, Dao Phuong, et al., "Robust control for buck converter based on optimization," 2017 International Conference on System Science and Engineering (ICSSE), Ho Chi Minh City, 2017, pp. 704-707.

[12] Quang N. H., et al., " Multi parametric model predictive control based on laguerre model for permanent magnet linear synchronous motors," International Journal of Electrical and Computer Engineering (IJECE), vol. 9, no. 2, pp. 1067-1077, 2019.

[13] El-Sousy, "Adaptive Dynamic Sliding Mode Control System using Recurrent RBFN for High-Performance Induction Motor Servo Drive," IEEE Transactions on Industrial Informatics, vol. 9, no. 4, pp. 1922-1936, 2013.

[14] Ziting Chen, Zhijun Li, Philip Khan, Akmeliawati, Bhatti, Khan, "Robust Stabilization of Underactuated nonlinear Systems: A fast terminal Sliding Mode Approach,” ISA Transactions, vol. 66, pp. 241-248, 2017.

[15] Clément Tréangle, Mondher Farza, Mohammed M. Saad, "Filtered high gain observer for a class of uncertain nonlinear systems with sampled outputs," Automatica, vol. 101, pp. 197-206, 2019.

[16] Dong Y., You L., Bing X., Wene L., "Robust finite-time adaptive control algorithm for satellite fast attitude maneuver," Journal of the Franklin Institute, vol. 357, no. 16, pp. 11558-11583, 2020.

[17] Mofid O., Mobayen S., "Adaptive sliding mode control for finite-time stability of quad-rotor UAVs with parametric uncertainties," ISA transactions, vol. 72, pp. 1-14, 2018.

[18] Sun T., Cheng L., Wang W., Pan Y., "Semiglobal exponential control of Euler-Lagrange systems using a slidingmode disturbance observer," Automatica, vol. 112, p. 108677, 2020.

[19] Yang Xiaowei, Jianyong Yao, Wenxiang Deng, "Output feedback adaptive super-twisting sliding mode control of hydraulic systems with disturbance compensation," ISA transactions, 2020. https://doi.org/10.1016/j.isatra.2020.09.014

[20] Fu C., Tian Y., Huang H., Zhang L., Peng C., "Finite-time trajectory tracking control for a 12-rotor unmanned aerial vehicle with input saturation," ISA transactions, vol. 81, pp. 52-62, 2018.

[21] Xiao L., Zhu Y., "Sliding mode output feedback control based on tracking error observer with disturbance estimator," ISA transactions, vol. 53, no. 4, pp. 1061-1072, 2014.

[22] Christofer Edwards, Yuri Shtessel, "Adaptive Continuous Higher Order Sliding Mode Control," Automatica, vol. 65, pp. 183-190, 2016.

[23] Maria Thomas, Kamal, Bandyopadhyay, Vachhani, "Continuous Higher Order Sliding Mode Control for a class of Uncertain MIMO nonlinear Systems: an ISS Approach,” European Journal of Control, vol. 41, pp. 1-7, 2018.

[24] Zheng Zhu, Xia Fu, "Adaptive Sliding Mode Control for Attitude Stabilization with Actuator Saturation," IEEE Transactions on Industrial Electronics, vol. 58, no. 10, pp. 4898-4907, 2011.

[25] Hassan K. Khalil, Laurent Praly, "High-gain observers in nonlinear feedback control," International Journal of Robust and Nonlinear Control, vol. 24, no. 6, pp. 993-1015, 2013.

[26] Ma L., Zong G., Zhao X., Huo X., "Observed-based adaptive finite-time tracking control for a class of nonstrict feedback nonlinear systems with input saturation," Journal of the Franklin Institute, vol. 357, no. 16, pp. 11518$11544,2019$.

[27] Huang Y., Liu Y., "A compact design scheme of adaptive output-feedback control for uncertain nonlinear systems," International Journal of Control, vol. 92, no. 2, pp. 261-269, 2019.

[28] Zeng T., Ren X., Zhang Y., "Fixed-time Sliding Mode Control and High-gain Nonlinearity Compensation for Multi-motor Driving System," IEEE Transactions on Industrial Informatics, vol. 16, no. 6, pp. 4090-4098, 2020.

[29] Inlin Wang, Xuemei Ren, and Qiang Chen, "Cascade Optimal Control for Tracking and Synchronizationof a Multimotor Driving System," IEEE Transactions on Control Systems Tecnology, vol. 27, no. 3, pp. 1376-1384, 2019.

[30] Nam D. P., Thanh P. T., Tinh T. X., Dat T. T., Van V. M., "High-Gain Observer Based Output Feedback Controller for a Two-Motor Drive System: A Separation Principle Approach," AETA 2017-Recent Advances in Electrical Engineering and Related Sciences: Theory and Application, vol 465, pp. 840-859, 2018. 